\title{
CUIDADOS DE ENFERMAGEM PRESTADOS À CRIANÇA HOSPITALIZADA COM DOR ONCOLÓGICA CRÔNICA: PERCEPÇÕES DOS PROFISSIONAIS DE SAÚDE
}

\author{
NURSING CARE TO HOSPITALIZED \\ CHILDREN WITH CHRONIC CANCER PAIN: \\ HEALTH PROFESSIONAL PERCEPTION
}

\section{CUIDADOS DE ENFERMERIA OFRECIDOS A LOS NIÑOS HOSPITALIZADOS CON DOLOR ONCOLÓGICO CRÓNICO: LA PERCEPCIÓN DE PROFESIONALES DE SALUD}

\author{
Thiago Privado da Silva ${ }^{1}$ \\ Laura Johanson da Silva ${ }^{2}$ \\ Italo Rodolfo Silva ${ }^{3}$ \\ Juliana Maria Rego Maciel Cardoso ${ }^{4}$ \\ Joséte Luzia Leite
}

Como citar este artigo: Silva TP, Silva LJ, Silva IR, Cardoso JMRM, Leite JL. Cuidados de enfermagem prestados à criança hospitalizada com dor oncológica crônica: percepções dos profissionais de saúde. Rev. baiana enferm. 2019;33:e29690

Objetivo: compreender a percepção dos profissionais de saúde sobre os cuidados de enfermagem prestados à criança hospitalizada com dor oncológica crônica. Método: abordagem qualitativa, ancorada nos referenciais teórico e metodológico, respectivamente, da Teoria da Complexidade e da Teoria Fundamentada em Dados. Os dados foram coletados por meio de entrevista semiestruturada e observação não participante. Participaram da pesquisa 21 profissionais de saúde. Resultados: os profissionais de saúde qualificaram como boa a assistência de enfermagem oferecida à criança com dor oncológica crônica. No entanto, foi revelada a necessidade de avanços: nos aspectos estruturais da unidade e organizacionais do cuidado, na abordagem ao familiar da criança, na abordagem à criança em precaução de contato e valorização profissional. Conclusão: o cuidado de enfermagem prestado à criança com dor oncológica crônica apresentava-se como um desafio, seja pelas necessidades de melhorias apontadas, seja por questões gerenciais e de liderança.

Descritores: Enfermagem Pediátrica. Cuidados de Enfermagem. Dor do Câncer. Hospitais. Pessoal de Saúde. Criança.

Objective: understanding the perception of health professionals with regards to the nursing care offered to children hospitalized with chronic cancer pain. Method: qualitative approach, anchored in theoretical and methodological frameworks from, respectively, the Theory of Complexity and Grounded Theory. Data were collected through a

\footnotetext{
Enfermeiro. Doutor em Enfermagem. Professor da Universidade Federal do Rio de Janeiro. Macaé, Rio de Janeiro, Brasil. thiagopsilva87@gmail.com

Enfermeira. Doutora em Enfermagem. Professor/a da Universidade Federal do Estado do Rio de Janeiro. Rio de Janeiro, Rio de Janeiro, Brasil.

Enfermeiro. Doutor em Enfermagem. Professor da Universidade Federal do Rio de Janeiro. Macaé, Rio de Janeiro, Brasil.

Enfermeira. Mestre em Enfermagem. Professora da Universidade Federal do Rio de Janeiro. Macaé, Rio de Janeiro, Brasil.

Pedagoga. Enfermeira. Doutora em Enfermagem. Professora da Universidade Federal do Estado do Rio de Janeiro. Rio de Janeiro, Rio de Janeiro, Brasil.
} 
semi-structured interview with non-participant observation. A total of 21 bealth professionals participated in the research. Results: the health professionals evaluated the nursing assistance offered to children with chronic cancer pain as good. However, the need for some improvements was found regarding the structural and organizational aspects of the unit, in the approach to the relatives of the child, in the approach to the child who require contact precautions, and valuing of the professionals. Conclusion: the nursing care offered to the child with chronic cancer pain was challenging, be it due to the improvement needs, be it due to managerial and leadership issues.

Descriptors: Pediatric Nursing. Nursing Care. Cancer Pain. Hospitals. Health Personnel. Child.

Objetivo: comprender la percepción de profesionales de salud sobre los cuidados de enfermería ofrecidos a niños hospitalizados con dolor oncológico crónico. Método: abordaje cualitativo, basado em el referencial teórico de la Teoría de la Complexidad y en el referencial metodológico del Muestreo Teórico. Se colectó a los datos por medio de entrevista semiestructurada y observación no-participante. Los participantes fueran 21 profesionales de salud. Resultados: los profesionales de salud cualificaron la asistencia de enfermería ofrecida a los niños en dolor oncológico crónico como buena. Sin embargo, se reveló la necesidad de mejorías: en los aspectos estructurales de la unidad y en los organizacionales de la atención, en el abordaje a los familiares del niño, en el abordaje a los niños bajo precauciones de contacto, y con respecto a la valorización de los profesionales. Conclusión: la atención en enfermería ofrecida a los niños con dolor oncológico crónico es un desafío, sea por las necesidades de mejorías indicadas o por los problemas administrativos y de liderazgo.

Descriptores: Enfermería Pediátrica. Atención de Enfermería. Dolor en Cáncer. Hospitales. Personal de Salud. Niño.

\section{Introdução}

O câncer em crianças e adolescentes de zero a 19 anos é considerado raro quando comparado ao câncer em adultos ${ }^{(1)}$. No Brasil, estimou-se que, em 2016, teria ocorrido 12.600 novos casos de câncer em crianças e adolescentes, considerando a faixa etária citada. Nessa conjuntura, as regiões Sudeste e Nordeste teriam apresentado os maiores números de casos novos, respectivamente, com 6.050 e 2.750, seguidas pela região Sul, com 1.320, região Centro-Oeste, com 1.270, e região Norte, com 1.210 novos casos ${ }^{(2)}$.

A magnitude da realidade exposta torna o câncer um importante problema de saúde pública, seja na infância, seja na vida adulta. No caso da criança, foco deste estudo, o câncer demanda, dos envolvidos no seu combate, investimentos financeiros e intelectuais na elaboração de projetos, programas e políticas públicas sociais e de saúde, qualificação profissional, fortalecimento de uma rede de cuidados especializados, iniciativas de prevenção e diagnóstico precoce, como também estratégias de cuidado que possibilitem promover a qualidade de vida dessas crianças. Nessa conjuntura, a dor destaca-se como um dos sintomas mais presentes, configurando-se como causa de sofrimento na criança e nos familiares, condicionando, desse modo, a qualidade de vida, a hospitalização e o tratamento antineoplásico ${ }^{(3-4)}$.

Reforçam o exposto, os resultados de uma pesquisa internacional ${ }^{(3)}$, a qual revelou que $52 \%$ das crianças com leucemia em tratamento de câncer apresentaram dor crônica ao longo do seu tratamento e os locais mais comuns de dor foram: costas, pernas e estômago $(8,0 \%)$ cada, peito (6,0\%), braços e cabeça (4,0\%), cada $^{(3)}$. Em consonância com esse resultado, uma outra pesquisa $^{(4)}$, realizada em hospitais na Nigéria, apresentou a dor como um dos principais sintomas vivenciados pela criança em tratamento de câncer.

A dor oncológica pode manifestar-se como aguda ou crônica. A dor aguda surge como resultado de uma lesão tecidual e tende a desaparecer quando o ferimento cicatriza. Por outro lado, a dor crônica é contínua (persistente) ou recorrente (episódica) e persiste além do tempo normal esperado ${ }^{(5)}$. Enquanto a dor aguda é geralmente autolimitada, em alguns pacientes, a dor persiste além do tempo de cura esperado (definido $>3-6$ meses) e evolui para um estado crônico ${ }^{(6)}$. 
Em relação à presença de dor crônica em crianças com doença crônica, tal qual o câncer, destaca-se a sua capacidade de afetar negativamente as múltiplas dimensões do ser criança, incluindo seu crescimento e desenvolvimento, a prática de atividades físicas, a frequência escolar, o padrão de sono, a interação familiar, as relações sociais, o humor, entre outros aspectos ${ }^{(5)}$.

Por essa razão, admite-se que o cuidado à criança hospitalizada com dor oncológica crônica é complexo, pois solicita do profissional de saúde um olhar que transcenda os aspectos físicos da dor, considerando o seu caráter multidimensional e seus efeitos na dinâmica familiar, a qual também é impactada por essa condição. No que tange ao cuidado profissional prestado a essa criança, desenvolvido pela equipe de enfermagem, revela-se de extrema importância e se dirige, sobremodo, para as ações de promoção de conforto e qualidade de vida, aspectos essenciais no tratamento da criança.

Nessa conjuntura, os impactos dos cuidados de enfermagem, desenvolvidos para atender as necessidades da criança e/ou do familiar, são percebidos por todos os envolvidos nas relações de cuidado. Assim, compreender a percepção dos profissionais de saúde sobre os cuidados de enfermagem à criança hospitalizada com dor oncológica crônica surge como uma importante possibilidade de qualificar a assistência de enfermagem, fato que favorece reconhecer as potencialidades, os avanços e também as fragilidades e limitações.

A compreensão subsidiada pela percepção profissional pode ajudar a equipe de enfermagem a traçar estratégias mantenedoras da qualidade assistencial, bem como a lidar com as marcas da complexidade no âmbito do planejamento das ações ${ }^{(7)}$ que permeiam as relações de cuidado, a saber: incertezas, desafios, imprevisibilidades e a condição multidimensional da dor oncológica crônica. É, portanto, uma possibilidade de avançar nos estudos sobre o tema, os quais têm se concentrado na avaliação e no manejo da dor oncológica ${ }^{(8-11)}$.

Desse modo, questiona-se: Como os profissionais de saúde percebem os cuidados de enfermagem prestados à criança hospitalizada com dor oncológica crônica? O objetivo desta pesquisa é compreender a percepção dos profissionais de saúde sobre os cuidados de enfermagem prestados à criança hospitalizada com dor oncológica crônica.

\section{Método}

Pesquisa de abordagem qualitativa, realizada com base no referencial metodológico da Grounded Theory (GT), em português, Teoria Fundamentada em Dados (TFD), e no referencial teórico da Teoria da Complexidade na perspectiva de Edgar Morin. Utilizou-se a Teoria Fundamentada em Dados com o propósito de compreender o fenômeno em tela, com base na elaboração de conceitos teóricos enraizados nas relações sociais dos participantes da pesquisa. Nessa direção, a Teoria da Complexidade revelou-se apropriada para a análise e discussão dos dados qualitativos, pois possibilitou valorizar a complexidade da dor, bem como as interações, a ordem e a desordem que permeiam a prática dos cuidados de enfermagem.

Os dados foram coletados entre agosto de 2014 e junho de 2015, por meio de entrevista semiestruturada e observação não participante, ambas realizadas com profissionais de saúde lotados na Unidade de Internação Pediátrica (UIP) de um hospital público que se apresentava como referência no tratamento de doenças hematológicas, localizado no município do Rio de Janeiro, Brasil. A Unidade de Internação Pediátrica abrigava 13 leitos, sendo um destinado para a criança em precaução de contato. Nesse contexto, os principais diagnósticos das crianças hospitalizadas eram: leucemia, linfoma e doença falciforme.

As entrevistas foram gravadas em áudio e tiveram uma duração média de 45 minutos. Foram realizadas nas dependências da própria UIP. A observação não participante foi realizada em cinco ocasiões, durante o período diurno, após a análise dos discursos dos entrevistados e totalizou 54 horas. Os conteúdos foram registrados em notas de observação e relacionavam-se às atitudes, ações e estratégias de interação percebidas para o trabalho em equipe e para o cuidado 
à criança hospitalizada com dor oncológica crônica, o que favoreceu o adensamento teórico das categorias e subcategorias construídas.

Inicialmente, participaram da pesquisa sete enfermeiros, compondo o primeiro grupo amostral. A análise das entrevistas realizadas com esses profissionais revelou que o processo de trabalho do enfermeiro no cuidado à criança hospitalizada com dor oncológica crônica é condicionado pelas ações da equipe multiprofissional de saúde, bem como revelou que o trabalho em equipe configura-se como estratégia de ação fundamental para a qualidade do cuidado de enfermagem. Por essa razão, considerando o recurso da amostragem teórica ${ }^{(12)}$ da TFD, entrevistas foram desenvolvidas com outros profissionais de saúde, a fim de melhor compreender o fenômeno exposto. Participaram do segundo grupo amostral sete técnicos de enfermagem. O terceiro e último grupo amostral foi composto por outros sete profissionais de saúde, sendo duas médicas, duas fisioterapeutas, uma assistente social, uma psicóloga e uma farmacêutica. Isso só é possível porque, com esse método, os dados são coletados e analisados concomitantemente.

Todos os participantes enquadraram-se nos critérios de inclusão: ter experiência mínima de um ano no cuidado à criança oncológica, e esse mesmo período de vinculação profissional à instituição. Foram excluídos os profissionais de saúde que estavam de licença ou de férias, totalizando 3 profissionais. No que concerne à composição da equipe de enfermagem, são 22 técnicos de enfermagem, 1 auxiliar de enfermagem e 7 enfermeiros. Por plantão, a equipe multiprofissional é composta por 2 a 3 médicos, 1 fisioterapeuta, 1 assistente social, 1 psicóloga e 1 farmacêutico.

A análise comparativa aplicada ao primeiro grupo amostral culminou em códigos que demonstravam a teia de interações entre enfermeiros, técnicos de enfermagem e demais profissionais de saúde, guiando a coleta de dados para os demais grupos amostrais. A questão que norteou as entrevistas com os profissionais de saúde foi: Conte-me como você percebe o cuidado de enfermagem prestado à criança hospitalizada com dor oncológica crônica.

A coleta de dados foi finalizada quando se obteve a saturação teórica ${ }^{(12)}$, momento em que os novos dados coletados já não estavam alterando em consistência e densidade teórica os conceitos construídos. A análise comparativa dos dados ocorreu mediante as etapas de codificações: aberta, axial e seletiva ${ }^{(12)}$. Na etapa aberta, os dados foram codificados linha por linha, gerando os códigos preliminares que, por sua vez, após serem agrupados por similaridades de sentido, deram origem aos códigos conceituais. Estes foram comparados entre si e organizados por similaridades, originando as categorias e subcategorias.

$\mathrm{Na}$ codificação axial, as categorias foram relacionadas entre si e entre suas subcategorias, a fim de determinar suas propriedades e dimensões. Nesse momento da análise, foi utilizada uma ferramenta analítica denominada de Modelo Paradigmático, que possibilitou a reunião/ ordenação/integração das categorias previamente elaboradas, favorecendo o surgimento do fenômeno central do estudo, ocorrido na etapa de codificação seletiva. Na aplicação do Modelo Paradigmático, as categorias são relacionadas ao fenômeno central, conforme os seguintes elementos: condição causal, condição contextual, condição interveniente, estratégia de ação/interação e consequência.

$\mathrm{Na}$ codificação seletiva, também foi realizada a validação dos resultados ${ }^{(12)}$. Tal processo ocorreu nos meses de setembro e outubro de 2016 e contou com a participação de cinco examinadores, sendo: três pesquisadores da área da Enfermagem, com expertise na Grounded Theory e/ou em pesquisa na área da Gerência em Enfermagem, e duas enfermeiras assistenciais do primeiro grupo amostral da presente pesquisa. Destaca-se que a seleção dos examinadores ocorreu por conveniência. Aliado ao processo de codificação, foram realizados memorandos e diagramas que auxiliaram a análise teórica dos dados ${ }^{(12)}$.

Neste artigo, aborda-se a categoria conceitual que, no emprego do modelo paradigmático, se 
configurou como consequência do fenômeno central - Gerenciamento do cuidado de enfermagem prestado à criança hospitalizada com dor oncológica crônica: uma experiência de múltiplas inter-ações -, uma vez que revela a percepção dos profissionais de saúde sobre os cuidados de enfermagem prestados à criança hospitalizada com dor oncológica crônica, incluindo fragilidades e potencialidades.

Salienta-se que a coleta de dados somente foi iniciada após a aprovação do estudo pelo Comitê de Ética em Pesquisa da instituição coparticipante, que ocorreu no dia 29/9/2014, sob o Parecer n⿳⺈ 355/14, e pelo Comitê de Ética em Pesquisa da instituição proponente, que ocorreu no dia 3/10/2014, sob o Parecer nº 816.736 e CAAE no 32795514.8.0000.5238. Todos os participantes assinaram o Termo de Consentimento Livre e Esclarecido (TCLE).

Considerando as recomendações da Resolução no 466/2012 do Conselho Nacional de Saúde do Ministério da Saúde, as falas dos enfermeiros estão identificadas pela letra E; as dos técnicos de enfermagem pela letra T; as das médicas pela letra $\mathrm{M}$; as da farmacêutica pelas letras FC; as da psicóloga pela letra P; as das fisioterapeutas pelas letras FS; e as da assistente social pelas letras AS. Todas estão seguidas por um algarismo que se refere à ordem das entrevistas em cada grupo amostral (E1, T1, M1...).

\section{Resultados}

Do total de 21 participantes da pesquisa, apenas 1 era do sexo masculino e integrou o primeiro grupo amostral. O tempo de atuação profissional na pediatria variou entre 1 e 16 anos, enquanto que o tempo de experiência profissional no cuidado à criança com câncer variou entre 1 e 13 anos.

Neste artigo, apresenta-se a categoria "Tecendo considerações sobre a prática do cuidado de enfermagem prestado à criança hospitalizada com dor oncológica crônica", a qual é constituída por duas subcategorias, a saber: Refletindo sobre a assistência prestada à criança hospitalizada com dor oncológica crônica e ao seu familiar e
Apresentando possibilidades gerenciais e assistenciais no cuidado à criança hospitalizada com dor oncológica crônica.

\section{Refletindo sobre a assistência prestada à criança bospitalizada com dor oncológica crônica e ao seu familiar}

Nesta subcategoria, os profissionais de enfermagem reconheceram que a assistência prestada à criança hospitalizada com dor oncológica crônica necessitava de avanços na gestão de medicamentos, recursos materiais e recursos humanos. A seguir, estão as falas que retratam essa situação:

A falta de medicamento, de material, de pessoal. Precisa melhorar. (E6).

Acho que poderia melhorar essa falta de profissionais, o que nos deixa sobrecarregados. Em se tratando de criança, você tem que ter profissionais bons, em quantidade suficiente. (T5).

Acho que precisa mudar a marca de alguns materiais que não são bons. As crianças com doenças hematológicas são ruins de acesso e, quando você consegue acesso, você o perde rápido, porque o jelco não é de boa qualidade. (T6).

A necessidade de avanços nos registros de enfermagem e nos cuidados prestados à criança em precaução de contato foram apontados pelos participantes. Ademais, foi reconhecida a necessidade de maior atenção ao familiar da criança hospitalizada.

\begin{abstract}
Eu acho que a parte de registro de enfermagem e a precaução de contato podem melhorar. É uma coisa que eu vejo que passa batido pela equipe. Por exemplo, vai instalar um soro na criança em precaução de contato, entra sem capote, sem luva, sem EPI [equipamento de proteção individual]. (E5).
\end{abstract}

Eu creio que têm coisas que precisam melhorar sim. Pelo meu plantão, eu acho que poderia dar uma atenção maior aos pais, ouvir os pais, mas são três técnicos para muitas crianças e fica difícil. (T1).

Nessa direção, foi sugerida a necessidade de novas instalações no contexto pediátrico e institucional. A falta de um Centro de Terapia Intensiva Pediátrica (CTIP) ganha merecido destaque em função da gravidade das crianças atendidas nessa instituição. Outrossim, as crianças em precaução de contato ficam totalmente restritas ao leito, sem condições de estabelecerem interações 
com seus pares. Algumas falas que representam essa conjuntura estão expostas a seguir:

A gente aqui não tem um CTI pediátrico e a gente transfere a criança para outro bospital, quando o seu estado agrava. (E1).

Acho que, por ser uma criança que tem a necessidade de uma vida social, ela fica muito tempo internada aqui, e eu acho que o ambiente deveria ser o mais favorável para a criança, porque as que estão em precaução de contato não saem do leito nem para ir ao banbeiro. Às vezes, ela só sai para tomar banho pela manhã, durante o dia. Ela não pode ficar circulando, e elas reclamam muito isso. (T4).

A estrutura física da unidade precisa melhorar. Eu acho que os leitos ficam muito próximos um do outro e não se tem muita privacidade. (E6).

Aqui na enfermaria, há um leito do lado do outro, e quando eu falo com uma mãe, muitas vezes, a outra já está respondendo. Você vai falar com uma criança, a outra já está brincando com ela. (P6).

A subcategoria revelou também que, mesmo vivenciando tais desordens, os profissionais de enfermagem qualificam sua assistência como boa e adequada.

Adequada é, porém muitas coisas a gente precisa melhorar. Mas o básico está adequado. (E2).

Eu vejo que os cuidados estão adequados. Nós temos falhas que são pontuais. Eu não tenho uma causa instalada, é uma coisa ou outra. Às vezes, é um detalhe que passa. Mas, no geral, eu vejo como boa a assistência. (E5).

Eu acho a assistência da minha equipe boa. (T1).

No âmbito do trabalho em equipe, outros profissionais de saúde reforçaram que a assistência de enfermagem prestado à criança hospitalizada com dor oncológica crônica tem sido boa, mesmo com tantos desafios a serem superados.

Aqui na pediatria, a equipe de enfermagem é ótima. Eles sabem quando a criança está com dor e precisa de uma medicação mais forte. Eles reavaliam a dor, aplicam a escala analógica de dor em todas as crianças internadas. A equipe é ótima! (M1).

Eu acho que o cuidado tem sido bom. A equipe de enfermagem é bem atenciosa com a criança. (FS4).

Os profissionais de enfermagem são os que mais interagem com a criança, quem mais tocam. Então, são muito importantes. Eu vejo que a equipe faz tudo o que pode. A maioria é atenciosa. Elas tentam interagir com a criança, tentam minimizar tudo o que ela [criança] está vivendo por conta da doença. (AS7).

Eu acho que a assistência tem sido boa [...] todos os profissionais trabalham realmente integrados na equipe multiprofissional de saúde. (P6).

\section{Apresentando possibilidades gerenciais e assistenciais no cuidado à criança bospitalizada com dor oncológica crônica}

Nesta subcategoria, os profissionais de enfermagem sinalizaram a possibilidade de intensificar a capacitação profissional para o desenvolvimento de analgesia não farmacológica, visando atenuar o sofrimento infantil e familiar.

Falta um pouquinho de autonomia para o enfermeiro desenvolver os tratamentos não farmacológicos. Mas isso requer preparo. A gente fica muito na base da avaliação da dor e na administração de medicamentos. (E7).

Acho que poderia ter outros meios sem ser a medicação, que pudessem ajudar essa criança, porque a utilização da medicação é muito grande. (T7).

A utilização de métodos de analgesia não farmacológica solicita conhecimento científico e capacitação profissional em todos os âmbitos e períodos de trabalho. Tal condição pode ser o ponto de partida para o gerenciamento de várias desordens identificadas, pois o profissional instrumentalizado pelo conhecimento é capaz de problematizar sua realidade e elaborar estratégias criativas para lidar com as inúmeras desordens que vivencia em seu processo de trabalho.

Eu acho muito interessante um treinamento mais adequado para os que estão chegando na instituição. (E2).

A capacitação dos profissionais de enfermagem que trabalham durante o serviço noturno, emergiu como um desafio para o serviço de educação continuada da instituição, haja vista a realidade apresentada pelos profissionais a seguir:

A maioria dos funcionários que trabalha à noite, também trabalha durante o dia e nem sempre é possivel ter um dia disponivel para vir para a instituição durante o dia fazer esses cursos. (T3).

Acho que, de noite, poderia vir uma equipe especializada para dar palestra dentro do próprio setor, para a gente não sair do posto e gerar deficiência de funcionário. Acho que isso facilitaria nosso aprendizado. (T5).

A valorização profissional foi pontuada como estratégia de enfrentamento do absenteísmo, bem como condição mantenedora da qualidade assistencial. Em que pese o sofrimento advindo do contexto oncológico pediátrico, é preciso pensar estratégias que tornem os profissionais 
de saúde motivados e satisfeitos no desenvolvimento do seu processo de trabalho.

A valorização profissional é um aspecto que merece aten-
ção, a fim de evitar ou reduzir o absenteísmo e favorecer
a motivação e a permanência do profissional em seu con-
texto de trabalho. Eu acho que deveria ter uma atenção
com os funcionários no momento de estresse [...] eu sinto
muita tristeza. (E2).

Acho que a instituição precisa melhorar a valorização com ofuncionário. (T3).

Alguns profissionais de saúde sugeriram que a estrutura física da Unidade de Internação Pediátrica poderia apresentar uma configuração mais lúdica e interativa, a fim de melhorar o acolhimento da criança hospitalizada.

Eu acho que o ambiente da criança em precaução de contato precisa melhorar, como o aquário carioca. Eles fizeram uma obra no aquário carioca e agora ele está lindo $e$ as crianças adoram. Acho que aqui na pediatria, deveria ter uma coisa para chamar a atenção da criança. (T4).

Vejo a necessidade de ter um espaço maior para a criança internada, porque nós temos uma salinha relativamente pequena para elas interagirem. As crianças externas, que vêm para quimioterapia, têm o aquário carioca. Mas a criança internada não tem esse tipo de espaço. A criança fica muito restrita ao leito. (FS5).

Diante do exposto, a categoria apontou os muitos desafios a superar e apresentou possibilidades gerenciais e assistenciais de oferecer, à criança hospitalizada com dor oncológica crônica, um ambiente mais acolhedor, humanizado e um cuidado de melhor qualidade.

\section{Discussão}

A categoria "Tecendo considerações sobre a prática do cuidado de enfermagem prestado à criança hospitalizada com dor oncológica crônica" apresentada neste artigo, possibilitou compreender que o cuidado de enfermagem prestado à criança hospitalizada com dor oncológica crônica enfrenta situações de ordem e desordem, necessitando de avanços no âmbito qualitativo e quantitativo, uma vez que possui fragilidades gerenciais e assistenciais que podem influenciar o seu desenvolvimento. Na lente da complexidade, a ordem e a desordem são dinâmicas e apresentam uma relação complementar, dialógica e não de oposição ou exclusão ${ }^{(7)}$.
A noção de ordem comporta as regularidades, estabilidades, constâncias, repetições, invariâncias e determinações. Por outro lado, a desordem envolve agitações, dispersões, turbulências, irregularidades, instabilidades, acidentes, acasos, ruídos, erros nos diferentes contextos da sociedade humana $^{(7)}$. A ordem e a desordem, por meio da relação dialógica que assumem, estão presentes e revelam-se necessárias no contexto dos cuidados de enfermagem, por possibilitarem avanços e inovações, por meio de (re)arranjos organizacionais e relacionais, conferindo flexibilidade e dinamismo ao processo de trabalho dos profissionais de enfermagem.

Os resultados sinalizam a necessidade de avanços no gerenciamento de medicamentos, recursos materiais e humanos. Em relação ao déficit de medicamentos, é importante destacar que sua ocorrência pode culminar em atrasos na sua administração, substituição por outros de menor potencial de alívio, conflitos interpessoais entre o profissional e o familiar e maior sofrimento da criança. A literatura ${ }^{(13)}$ reforça que o déficit de recursos materiais na instituição hospitalar pode configurar-se como fator limitador da prática do gerenciamento do cuidado de enfermagem prestado à criança hospitalizada em condição crônica.

Nessa conjuntura, ressalta-se que o desempenho das ações da equipe de enfermagem, nos diferentes contextos de atuação, sofre influência direta do quantitativo de recursos humanos disponíveis $^{(14)}$. Uma revisão integrativa ${ }^{(15)}$ qualificou o dimensionamento de pessoal de enfermagem como importante instrumento de suporte para o enfermeiro prover o qualitativo e o quantitativo de trabalhadores em seu contexto de trabalhado, configurando-se, desse modo, como uma importante estratégia de ação para minimizar os riscos e as consequências do absenteísmo na enfermagem.

Além disso, foi pontuada a necessidade de avanços nos registros de enfermagem. Uma pesquisa $^{(16)}$ identificou que foi frequente a falta do registro de enfermagem quanto ao manejo da dor oncológica, deixando dúvidas se ele foi ou não implementado, o medicamento utilizado 
e sua resolubilidade. No âmbito internacional, também se observam falhas no registro da dor, situação que pode colocar em risco a segurança do paciente $e^{(17-18)}$.

$O$ registro de enfermagem requer um olhar sob a perspectiva da ética profissional, uma vez que consta, na Resolução n⿳o 564/2017 ${ }^{(19)}$, do Conselho Federal de Enfermagem (COFEN), art. 36, o dever de o profissional de enfermagem registrar, no prontuário e em outros documentos, as informações inerentes e indispensáveis ao processo de cuidar, de modo claro, objetivo, cronológico, legível, completo e sem rasuras. No art. 86, consta a proibição de registrar informações incompletas e imprecisas sobre a assistência de enfermagem prestada. Para além dessa normativa, na prática de cuidados, considera-se importante as relações gerenciais, os recursos humanos e a capacitação, pois são condições que também podem influenciar a prática do registro profissional.

O cuidado de enfermagem prestado à criança hospitalizada com dor oncológica crônica deve considerar as demandas do familiar, já que ele também é afetado pela condição crônica da criança. Nessa conjuntura, ressalta-se que a relação entre o profissional de saúde e o familiar deve ser permeada pela atenção, empatia e pelo respeito mútuo. Portanto, o fortalecimento dessa relação no contexto hospitalar apresenta-se como algo desejável no planejamento da assistência. Sob essa perspectiva, um estudo identificou que a equipe de enfermagem reconheceu a necessidade de investir no relacionamento com o familiar da criança hospitalizada em condição crônica, por considerar importante a sua presença e participação no tratamento da criança ${ }^{(20)}$.

A família desempenha papel importante no crescimento e desenvolvimento infantil, o que significa que ela exerce uma importante influência no comportamento da criança. Na lente da complexidade ${ }^{(7)}$, entende-se que a família pode configurar-se tanto um espaço seguro quanto uma prisão para a criança. No contexto hospitalar, salienta-se que o familiar pode agir como um facilitador das relações interpessoais entre o profissional de saúde e a criança. Desse modo, ele pode favorecer a adesão da criança ao tratamento, tornar a experiência da hospitalização menos traumática e conferir mais segurança e confiança à criança nos momentos de sofrimento, dor e tensão. Portanto, a valorização do familiar no âmbito hospitalar apresenta-se como estratégia de ação necessária para a continuidade e qualidade dos cuidados de enfermagem.

A atuação profissional junto à família da criança hospitalizada surge como um desafio nas relações de cuidado. Nessa conjuntura, a literatura reforça o exposto, ao revelar que, não obstante a promulgação do Estatuto da Criança e do Adolescente e a importância do Cuidado Centrado na Família no contexto pediátrico, os enfermeiros parecem não compreender a importância do cuidado à família, tampouco desenvolveram habilidades para reorganizar o seu processo de trabalho diante da ampliação de sua unidade de cuidado ${ }^{(21)}$. Este fato imprime dificuldades nas relações de cuidado estabelecidas com a família da criança hospitalizada.

A valorização profissional foi pontuada como importante condição a ser considerada pelos gestores da unidade, pois é um fator que condiciona o significado que o trabalhador atribui ao seu processo de trabalho. Por esse olhar, compreende-se que tal condição tem relação proporcional com o sentimento de satisfação profissional, produtividade e alta qualidade do serviço prestado $^{(13)}$.

$\mathrm{Na}$ complexidade dos fenômenos sociais, é preciso considerar a relação do todo e das partes $^{(7)}$. Entende-se que o profissional de saúde, enquanto unidade autônoma, integra um todo, o qual pode ser a sua equipe ou a própria instituição. Esse elo complexo entre o todo e suas partes merece atenção, pois, do ponto de vista da complexidade, é compreensível a necessidade de a instituição oferecer o suporte ao profissional, para só assim poder contar com ele, por meio de sua atividade laboral. Existe, portanto, uma relação de retroatividade, na qual a unidade influencia e compõe o todo. Igualmente, o todo influencia e é composto por suas unidades ${ }^{(7)}$.

Ademais, destaca-se o espaço destinado à criança em precaução de contato, o qual 
limita-se ao leito por razões estruturais da unidade. Sobre tal circunstância, a literatura destaca que a precaução de contato em crianças imunocomprometidas, como é o caso de crianças em tratamento de câncer, apresenta-se como uma importante medida de prevenção de transmissão de infecção cruzada entre elas, como também entre seus familiares e acompanhantes ${ }^{(22)}$.

Embora os profissionais de enfermagem reconheçam a necessidade de avançar nos cuidados prestados à criança hospitalizada com dor oncológica crônica, nesta pesquisa eles qualificaram sua assistência como boa e adequada. De modo semelhante, os resultados demonstraram que outros profissionais de saúde também percebiam como boa a assistência de enfermagem prestada à criança hospitalizada com dor oncológica crônica, em especial pela atuação dos profissionais de enfermagem na avaliação e no manejo da dor, bem como por sua atenção com a criança.

Pontuou-se a necessidade de ampliar o investimento na capacitação profissional, sobremodo para o desenvolvimento de analgesia não farmacológica, posto que há ocasiões de déficit de medicamentos na instituição. No entanto, ressalta-se que a analgesia não farmacológica deve ser empregada de forma complementar à analgesia farmacológica, e não como uma substituta diante do déficit de medicamentos. Maior investimento nos métodos de analgesia não farmacológica pode contribuir para a redução do sofrimento infantil e familiar, favorecendo também a redução de custos do tratamento ${ }^{(5)}$.

Por esse olhar, admite-se que a capacitação dos profissionais de enfermagem para o manejo não farmacológico da dor oncológica crônica pode conferir maior autonomia, no âmbito do trabalho em equipe, facilitando a tomada de decisão e as relações de cuidado. Entretanto, cumpre destacar, com base no referencial da complexidade, que a autonomia a que se refere o presente artigo, não é a de uma liberdade absoluta, desprovida de qualquer dependência ${ }^{(7)}$, mas uma autonomia profissional, que reconhece sua dependência em relação aos elementos contextuais do cuidado multiprofissional de saúde.
Além de realizar o manejo da dor oncológica crônica, os profissionais de enfermagem devem ser capacitados pela instituição para atuar com segurança diante das especificidades do contexto oncológico pediátrico. Diante dessa consideração, ressalta-se o importante papel que o enfermeiro ocupa em favorecer o trabalho de toda a sua equipe no setor oncológico, a fim de garantir qualidade na assistência, que deve ser desenvolvida com base em aprimoramento técnico e científico, motivado e proporcionado pelo próprio enfermeiro ou pela instituição ${ }^{(23)}$.

Um dos desafios revelados no presente estudo consistiu em promover a capacitação dos profissionais de enfermagem que trabalhavam no serviço noturno. Os cursos oferecidos pela instituição ocorriam durante os serviços diurnos e, nesse período, muitos profissionais que trabalhavam no serviço noturno apresentavam outros vínculos de trabalho que os impossibilitavam de frequentar os cursos em outro período. Salienta-se a relevância da capacitação dos profissionais de enfermagem que trabalham no serviço noturno, pois uma pesquisa ${ }^{(24)}$ identificou que o perfil dos pacientes em atenção paliativa oncológica foi caracterizado como fator que aumenta a probabilidade de intercorrências à noite. Nessa conjuntura, surge a necessidade de a equipe de enfermagem, sobretudo o enfermeiro, saber intervir rapidamente para gerenciar as desordens que envolvem o ser humano e a sua finitude.

A estrutura física do contexto pediátrico esteve presente na avaliação dos participantes do estudo como espaço que precisa apresentar um aspecto visual mais suavizado e agradável à criança oncológica, tal como é o aquário carioca, o qual foi configurado de modo a oferecer à criança, em qualquer fase de seu desenvolvimento, atividades lúdicas de humanização, distração, relaxamento e segurança ${ }^{(25)}$.

Entre as contribuições deste estudo, destaca-se a necessidade de gerenciar o cuidado, levando em conta alguns fatores: importância da educação permanente no apoio ao gerenciamento da dor oncológica crônica e no seu correto registro no prontuário; necessidade de habilidades relacionais para se trabalhar com a 
família da criança hospitalizada e com a equipe multiprofissional de saúde; suporte institucional efetivo, com materiais e recursos humanos adequados, além de estratégias de valorização profissional. O estudo também revela a importância de um ambiente acolhedor no contexto intra-hospitalar para a criança que se encontra hospitalizada.

É importante ressaltar que este estudo apresenta limitações quanto ao critério de generalização teórica, haja vista que, a priori, os resultados não são capazes de representar outras realidades, o que aponta para a necessidade de novas pesquisas teóricas sobre objeto de pesquisa delimitado.

\section{Conclusão}

O exposto permitiu concluir-se que o cuidado de enfermagem prestado à criança hospitalizada com dor oncológica crônica apresentava-se como um desafio, seja pelas necessidades de melhorias apontadas, seja por questões gerenciais e de liderança. Entre as desordens mencionadas, constam o déficit de medicamentos, de recursos materiais e humanos.

O registro das ações desenvolvidas pelos profissionais de enfermagem na avaliação e no manejo da dor oncológica crônica necessita de avanços, especialmente por se tratar de uma importante questão ética. Do mesmo modo, foi compreendido que a abordagem profissional ao familiar e à criança em precaução de contato precisa ser aperfeiçoada, a fim de conferir uma atenção mais humanizada. Nesse sentido, destacou-se a necessidade de um ambiente mais acolhedor para a criança oncológica.

Investimento na qualificação profissional, sobretudo para desenvolver estratégias de analgesia não farmacológica, pautadas em evidências científicas e na segurança e autonomia profissional, foi revelado pelos participantes, em especial pelos profissionais de enfermagem, como um indicativo para melhor lidar com as desordens vivenciadas. Tal atividade pode facilitar uma segura tomada de decisão, favorecendo a resolução de algumas dificuldades manifestadas.

\section{Colaborações:}

1 - projeto, análise e interpretação dos dados: Thiago Privado da Silva, Laura Johanson da Silva, Italo Rodolfo Silva e Joséte Luzia Leite; 2 - redação do artigo e revisão crítica relevante do conteúdo intelectual: Thiago Privado da Silva, Laura Johanson da Silva, Italo Rodolfo Silva e Julia Maria Rego Maciel Cardoso; 3 - aprovação final da versão a ser publicada: Thiago Privado da Silva, Laura Johanson da Silva, Italo Rodolfo Silva e Julia Maria Rego Maciel Cardoso.

\section{Financiamento:}

Coordenação de Aperfeiçoamento de Pessoal de Nível Superior (CAPES).

\section{Referências}

1. Instituto Nacional de Câncer José Alencar Gomes da Silva. Incidência, mortalidade, morbidade hospitalar por câncer em crianças, adolescentes e adultos jovens no Brasil: informações dos registros de câncer e do sistema de mortalidade [Internet]. Rio de Janeiro (RJ); 2016 [cited 2019 Feb 23]. Available from: http://www1.inca.gov.br/ inca/Arquivos/incidencia-mortalidade-morbidadehospitalar-por-cancer.pdf

2. Instituto Nacional de Câncer José Alencar Gomes da Silva. Protocolo de diagnóstico precoce para oncologia pediátrica [Internet]. Rio de Janeiro (RJ); 2017 [cited 2019 Feb 20]. Available from: http:// bvsms.saude.gov.br/bvs/publicacoes/protocolo_ diagnostico_precoce_cancer_pediatrico.pdf

3. Fortier MA, Wahi A, Bruce C, Maurer EL, Stevenson R. Pain Management at home in children with cancer: a daily diary study. Pediatr Blood Cancer [Internet]. 2014 [cited 2019 Feb 22];35(2):115-25. Available from: https://www.ncbi.nlm.nih.gov/ pubmed/24376073

4. Olagunju AT, Sarimiye FO, Olagunju TO, Habeedu MYM, Aina OF. Child's symptom burden and depressive symptoms among caregivers of children with cancers: an argument for early integration of pediatric palliative care. Ann Palliat Med [Internet]. 2016 [cited 2019 Sep 8];5(3):157-65. Available from: https://www.ncbi.nlm.nih.gov/ pubmed/27199271 
5. World Health Organization. WHO guidlines on the pharmacological treatment of persisting pain in children with medical illnesses [Internet]. Geneva; 2012 [cited 2019 Feb 22]. Available from: http://whqlibdoc.who.int/ publications/2012/9789241548120_Guidelines.pdf

6. American Pain Society. Assessment and management of children with chronic pain [Internet]. Chicago, IL; 2012 [cited 2019 Feb 22]. Available from: http://www.americanpainsociety. org/uploads/pdfs/aps12-pcp.pdf

7. Morin E. Introdução ao pensamento complexo. 4a ed. Porto Alegre (RS): Sulina; 2015.

8. Gauhar Afshan FCPS, Khalid Bashir MCPS. Cancer pain in children: a two-step strategy. Anaesth, Pain Intensive Care [Internet]. 2014 [cited 2019 Feb 22];18(1):106-10. Available from: http://www. apicareonline.com/special-article-cancer-pain-inchildren-a-two-step-strategy/

9. Batalha LMC, Fernandes AM, Campos C, Gonçalves AMPMPC. Pain assessment in children with cancer: a systematic review. J Nurs Referência [Internet]. 2015 Apr/May/Jun [cited 2019 Feb 22];4(5):119-27. Available from: file://C:/Users/User/Downloads/ 13_Rev._Enf._Ref._RIV14013ARS_english.pdf

10. Aziznejadroshan P, Alhani F, Mohammadi E. Experiences of Iranian nurses on the facilitators of pain management in children: a qualitative study. Pain Res Treat. 2016;2016(6). DOI: http://dx.doi. org/10.1155/2016/3594240

11. Wiffen PJ, Cooper TA, Anderson AK, Gary AL, Gregóire MC, Ljungman G, et al. Opioids for cancer-related pain in children and adolescents. Cochrane Database Syst Rev. 2017 Jul;7:CD012564. doi: 10.1002/14651858.CD012564.pub2

12. Strauss AL, Corbin J. Pesquisa qualitativa: técnicas e procedimentos para o desenvolvimento de teoria fundamentada. 2a ed. Porto Alegre (RS): Artmed; 2008

13. Silva TP, Silva IR, Leite JL. Interactions in the management of nursing care to hospitalized children with chronic conditions. Texto Contexto Enferm. 2016;25(2):e1980015. DOI: http://dx.doi. org/10.1590/0104-07072016001980015

14. Formenton F, Mininel VA, Laus AM. Absenteísmo por doença na equipe de enfermagem de uma operadora de plano de saúde. Rev Enferm UERJ [Internet]. 2014 [cited 2019 Feb 2012];22(1):42-9. Available from: http://www.e-publicacoes. uerj.br/index.php/enfermagemuerj/article/ view/11416/8976

15. Martinato MCNB, Severo DF, Marchand EAA, Siqueira HCH. Absenteísmo na enfermagem: uma revisão integrativa. Rev Gaúch Enferm [Internet]. 2010 [cited 2019 Feb 2012];31(1):160-6. Available from: http://www.scielo.br/scielo.php?script=sci_ arttext\&pid=S1983-14472010000100022

16. Bueno PC, Neves ET, Rigon AG. Manejo da dor em crianças com câncer: contribuições para a enfermagem. Cogitare Enferm [Internet]. 2011 [cited 2019 Feb 2012];16(2):226-31. Available from: http://revistas.ufpr.br/cogitare/article/viewFile/ 20307/14208

17. Song W, Eaton LH, Gordon DB, Hoyle C, Doorenbos AZ. Evaluation of evidence-based nursing pain management practice. Pain Manag Nurs [Internet]. 2015 [cited 2019 Jun 8];16(4):45663. Available from: https://www.ncbi.nlm.nih.gov/ pmc/articles/PMC4531385/\#R10

18. Unné A, Rosegren K. Staff experience of pain management: an improvement in palliative care. Pharmacy [Internet]. 2013 [cited 2019 Jun 8];1(2):119-36. Available from: http://www.mdpi. com/2226-4787/1/2/119

19. Conselho Federal de Enfermagem. Resolução COFEN no 564/2017. Aprova o novo Código de Ética dos Profissionais de Enfermagem. Brasília; 2017.

20. Silva TP, Silva MM, Alcantara LM, Silva IR, Leite JL. Establishing action/interaction strategies for care delivery to hospitalized children with chronic conditions. Esc Anna Nery [Internet]. 2015 Apr/ Jun [cited 2019 Feb 22];19(2):279-85. Available from: http://www.scielo.br/scielo.php?script=sci_ arttext\&pid=\$1414-81452015000200279

21. Corrêa AR, Andrade AC, Manzo BF, Couto DL, Duarte ED. As práticas do Cuidado Centrado na Família na perspectiva do enfermeiro da Unidade Neonatal. Esc Anna Nery [Internet]. 2015 out/ dez [cited 2019 Feb 22];19(4):629-34. Available from: http://www.scielo.br/pdf/ean/v19n4/14148145-ean-19-04-0629.pdf

22. Azevedo PMC, Souza TP, Almeida CPB. Prevenção de infecção hospitalar em unidades de internação pediátrica: uma revisão da literatura. Rev Saúde Com [Internet]. 2016 [cited 2019 Feb 22];12(3):65665. Available from: http://www.uesb.br/revista/ Rsc/ojs/index.php/rsc/article/viewFile/348/390 
23. Santos FC, Camelo SHH, Laus AM, Leal LA. O enfermeiro que atua em unidades hospitalares oncológicas: perfil e capacitação profissional. Enferm glob [Internet]. 2015 [cited 2019 Feb 22];14(38):313-24. Available from: http://scielo. isciii.es/pdf/eg/v14n38/pt_revision3.pdf

24. Silva MM, Moreira MC, Leite JL, Erdmann AL. O trabalho noturno da enfermagem no cuidado paliativo oncológico. Rev Latino-Am Enferm [Internet]. 2013 [cited 2019 Feb 22];21(3):773-9. Available from: http://www.scielo.br/pdf/rlae/ v21n3/pt_0104-1169-rlae-21-03-0773.pdf
25. Gomes IP, Collet N, Reis PED. Ambulatório de quimioterapia pediátrica: a experiência no aquário carioca. Texto Contexto Enferm [Internet]. 2011 [cited 2019 Feb 22];20(3):585-91. Available from: http://www.scielo.br/scielo.php?script $=$ sci_ arttext\&pid=S0104-07072011000300021

Recebido: 25 de fevereiro de 2019

Aprovado: 9 de setembro de 2019

Publicado: 14 de novembro de 2019

A Revista Baiana de Enfermagem utiliza a Licença Creative Commons - Atribuição-NãoComercial 4.0 Internacional.

https://creativecommons.org/licenses/by-nc/4.0/

Este artigo é de acesso aberto distribuído sob os termos da Licença Creative Commons (CC BY-NC).

Esta licença permite que outros remixem, adaptem e criem a partir do seu trabalho para fins não comerciais. Embora os novos trabalhos tenham de lhe atribuir o devido crédito e não possam ser usados para fins comerciais, os usuários não têm de licenciar esses trabalhos derivados sob os mesmos termos. 